\title{
Prostate cancer-associated mutations in speckle-type POZ protein (SPOP) regulate steroid receptor coactivator 3 protein turnover
}

\author{
Chuandong Geng ${ }^{\mathrm{a}, \mathrm{b}}$, Bin He ${ }^{\mathrm{a}, \mathrm{b}}$, Limei $\mathrm{Xu}^{\mathrm{c}}$, Christopher E. Barbieri ${ }^{\mathrm{c}, \mathrm{d}}$, Vijay Kumar Eedunurie, Sue Anne Chew ${ }^{\mathrm{a}, \mathrm{b}}$, \\ Martin Zimmermann $^{a, b}$, Richard Bond ${ }^{a, b}$, John Shou ${ }^{a, b}$, Chao Li ${ }^{b}$, Mirjam Blattner ${ }^{c}$, David M. Lonard ${ }^{b}$, \\ Francesca Demichelis ${ }^{\mathrm{f}, \mathrm{g}}$, Cristian Coarfa ${ }^{\mathrm{b}}$, Mark A. Rubin ${ }^{\mathrm{c}, \mathrm{d}, \mathrm{h}}$, Pengbo Zhou', Bert W. O'Malley ${ }^{\mathrm{b}, \mathrm{i}, 1}$, \\ and Nicholas Mitsiades ${ }^{a, b, i, i}$
}

Departments of a Medicine and ${ }^{b}$ Molecular and Cellular Biology and 'Center for Drug Discovery, Baylor College of Medicine, Houston, TX 77030; 'Department of Pathology and Laboratory Medicine, ${ }^{\mathrm{d} B r a d y}$ Urologic Foundation, Department of Urology, and ${ }^{\mathrm{h}}$ Institute for Precision Medicine, Weill Cornell Medical College of Cornell University and New York Presbyterian Hospital, New York, NY 10065; ${ }^{\mathrm{e}}$ Adrienne Helis Malvin Medical Research Foundation, New Orleans, LA 70130; 'Centre for Integrative Biology, University of Trento, Povo, 38123 Trento, Italy; and Institute for Computational Biomedicine, Department of Physiology and Biophysics, Weill Cornell Medical College of Cornell University, New York, NY 10065

Contributed by Bert W. O’Malley, March 11, 2013 (sent for review February 6, 2013)

The p160 steroid receptor coactivators (SRCS) SRC-1, SRC-2 [nuclear receptor coactivator (NCOA)2], and SRC-3 [amplified in breast cancer 1 (AIB1)/NCOA3] are key pleiotropic "master regulators" of transcription factor activity necessary for cancer cell proliferation, survival, metabolism, and metastasis. SRC overexpression and overactivation occur in numerous human cancers and are associated with poor clinical outcomes and resistance to therapy. In prostate cancer (PC), the p160 SRCs play critical roles in androgen receptor transcriptional activity, cell proliferation, and resistance to androgen deprivation therapy. We recently demonstrated that the E3 ubiquitin ligase adaptor speckle-type poxvirus and zinc finger (POZ) domain protein (SPOP) interacts directly with SRC-3 and promotes its cullin 3-dependent ubiquitination and proteolysis in breast cancer, thus functioning as a potential tumor suppressor. Interestingly, somatic heterozygous missense mutations in the SPOP substrate-binding cleft recently were identified in up to $15 \%$ of human PCs (making SPOP the gene most commonly affected by nonsynonymous point mutations in $\mathrm{PC}$ ), but their contribution to $\mathrm{PC}$ pathophysiology remains unknown. We now report that PC-associated SPOP mutants cannot interact with SRC-3 protein or promote its ubiquitination and degradation. Our data suggest that wild-type SPOP plays a critical tumor suppressor role in PC cells, promoting the turnover of SRC-3 protein and suppressing androgen receptor transcriptional activity. This tumor suppressor effect is abrogated by the PC-associated SPOP mutations. These studies provide a possible explanation for the role of SPOP mutations in PC, and highlight the potential of SRC-3 as a therapeutic target in PC.

proteasome | MATH domain | BTB domain

Drostate adenocarcinoma (PC) arises as an androgen-sensitive, androgen receptor (AR)-dependent malignancy, as highlighted by the clinical anticancer activity of gonadal androgen suppression and of recently approved agents such as the androgen synthesis inhibitor abiraterone (1) and the AR antagonist enzalutamide (2). The importance of the AR axis in PC pathophysiology is further illustrated by the frequent overexpression in PC, especially after exposure to androgen deprivation therapy, of enzymes involved in androgen synthesis (3), as well as AR itself (3) and AR coactivators $(4,5)$.

The p160 steroid receptor coactivators (SRCs) SRC-1, SRC-2 (also known as TIF2, GRIP1, and NCOA2), and SRC-3 (also known as AIB1, ACTR, and NCOA3) are key pleiotropic "master regulators" of transcription factor activity necessary for cancer cell proliferation, survival, metabolism, and metastasis (6). SRC overexpression and overactivation occur in numerous human cancers due to a variety of genomic, transcriptional, and posttranslational mechanisms, and are associated with poor clinical outcomes and resistance to therapy, suggesting that the SRCs are important therapeutic targets (6). In PC, the p160 SRCs play critical roles in AR transcriptional activity, cell proliferation, migration, and resistance to androgen deprivation therapy (7-9). Depletion of p160 SRCs in hormone-dependent PC and in CRPC cell lines impedes cell proliferation and AR transcriptional activity, including that of the AR-dependent induction of TMPRSS2-ERG (4, 6, 10-13). Elevated expression of all three p160 SRCs occurs in PC and is associated with a shorter time to recurrence and overall more aggressive disease $(4,12)$. Gene amplification, point mutations, and widespread overexpression of SRC-2 (NCOA2) have been reported in PC and are associated with increased AR transcriptional activity and inferior clinical outcomes (5).

However, the mechanisms underlying the overexpression of SRC-3 protein in PC are less well defined, especially as SRC-3 gene amplifications or mRNA overexpression are not commonly encountered in PC $(5,14,15)$. Instead, it is possible that SRC-3 expression could be regulated posttranslationally.

We recently demonstrated that the E3 ubiquitin ligase adaptor speckle-type POZ protein (SPOP) interacts directly with SRC-3 and promotes its cullin 3 (Cul3)-dependent ubiquitination and proteolysis in breast cancer, thus functioning as a potential tumor suppressor (16). SPOP contains two conserved domains: an N-terminal MATH (Meprin and Traf Homology) domain that recruits substrate proteins, and a C-terminal BTB (Bric-a-bracTramtrack-Broad complex) domain that interacts with Cul3 (17, 18). Somatic heterozygous missense SPOP mutations, clustered in the MATH domain (Fig. $1 A$ ), were recently identified in 6$15 \%$ of PCs (19-21), but their contribution to PC pathophysiology remains unknown.

In the present study, we wished to determine the impact of wildtype (WT) and mutant (mt) SPOP on SRC-3 protein turnover and AR transcriptional activity. Our data suggest that SPOP ${ }^{\mathrm{WT}}$ plays a critical tumor suppressor role in PC cells by promoting the turnover of SRC-3 protein and, thus, suppressing AR transcriptional activity. This tumor suppressor effect is abrogated by the PC-associated SPOP mutations. These studies provide a possible

Author contributions: C.G., B.H., C.E.B., M.A.R., P.Z., B.W.O., and N.M. designed research; C.G., B.H., L.X., V.K.E., S.A.C., M.Z., R.B., and J.S. performed research; C.L. contributed new reagents/analytic tools; C.G., L.X., C.E.B., M.B., D.M.L., F.D., C.C., M.A.R., P.Z., B.W.O., and N.M. analyzed data; and C.G. and N.M. wrote the paper.

The authors declare no conflict of interest.

${ }^{1}$ To whom correspondence may be addressed. E-mail: berto@bcm.edu or mitsiade@bcm. edu.

This article contains supporting information online at www.pnas.org/lookup/suppl/doi:10. 1073/pnas.1304502110/-/DCSupplemental. 

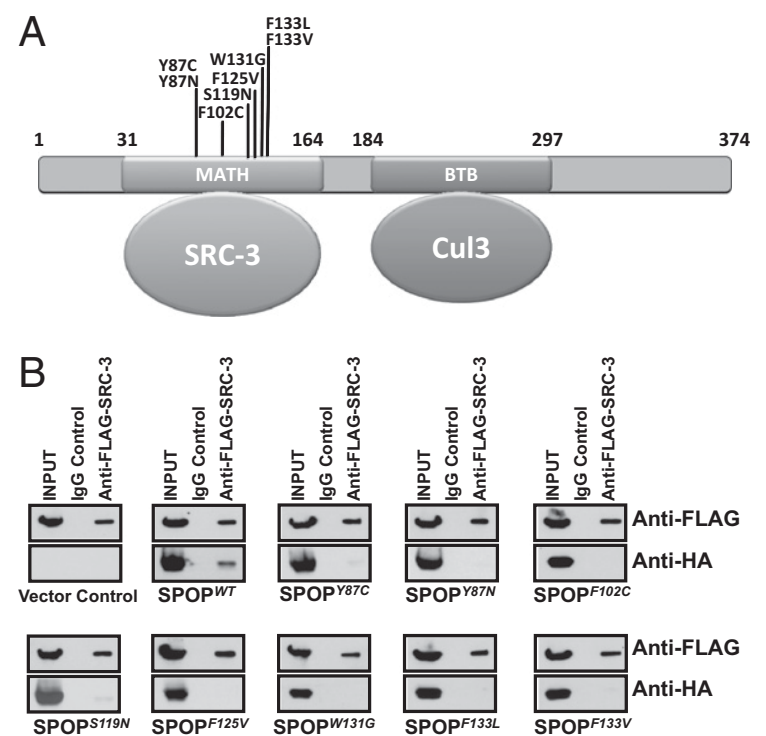

Fig. 1. $P C$-associated SPOP mutants lack the capacity of SPOPWT to interact with SRC-3. (A) Distribution on the SPOP gene of the most common mutations found in PC. These recurrent mutations are clustered to the $\mathrm{N}$-terminal MATH domain, which is necessary for SPOP binding to substrates (including SRC-3). (B) HEK293T cells were transfected with pCDNA3.1-HA-SPOP (WT or each individual mutant; described in Materials and Methods) or FLAG-tagged SRC-3 expression vectors for $2 \mathrm{~d}$. Cell lysates containing approximately equal amounts of each expressed HA-SPOP (WT or mutant) and SRC-3 protein were mixed overnight at $4{ }^{\circ} \mathrm{C}$ and immunoprecipitated with antiFLAG M2 antibody. SDS/PAGE and immunoblotting were used to detect HA-SPOPs and FLAG-SRC-3. The input was loaded at $1 / 10$ of the total lysate amount subjected to each immunoprecipitation experiment. SPOPWT was immunoprecipitated together with FLAG-tagged SRC-3. This interaction between SPOP and SRC-3 was attenuated in the case of the PC-associated SPOP mutants.

explanation for the role of SPOP mutations in PC, and highlight the potential of SRC-3 as a therapeutic target in PC.

\section{Results}

PC-Associated SPOP Mutants Lack the Capacity of SPOPWT to Interact with SRC-3. Because the PC-associated SPOP mutations cluster in the substrate-binding MATH domain (Fig. 1A), we hypothesized that they interfere with the capacity of SPOP to recruit SRC-3. Using coimmunoprecipitation (co-IP) in transiently transfected HEK293T cells, we confirmed that SPOP ${ }^{\mathrm{WT}}$ can be immunoprecipitated together with FLAG-tagged SRC-3 (Fig. 1B), but not SRC-1 or SRC-2 (Fig. S1 $A$ ). The interaction between SPOP and SRC- 3 was attenuated in the case of the PC-associated SPOP mutants (Fig. 1B).

PC-Associated SPOP Mutants Lack the Capacity of SPOPWT to Promote SRC-3 Protein Turnover. We next examined the capacity of PCassociated SPOP mutants to promote degradation of SRC-3 protein. In agreement with our previous report (16), exogenous expression of SPOP ${ }^{\mathrm{WT}}$ in HEK293T cells efficiently promoted degradation of SRC-3 protein in a dose-dependent manner (Fig. $2 A$ ). Furthermore, we also documented that this action of SPOP is unique to SRC-3, as SPOP ${ }^{\mathrm{WT}}$ did not promote degradation of the other two p160 SRC family members, SRC-1 or SRC-2 (Fig. S1B). However, none of the PC-associated SPOP mutants could promote degradation of SRC-3 protein (Fig. $2 A$ ). Whereas certain SPOP mutants induced no change in SRC-3 protein levels (loss-of-function effect), other SPOP mutants (such as F102C, F125V, and W131G) increased SRC-3 protein levels above baseline (i.e., no exogenous
SPOP) levels, suggesting a possible gain-of-function "dominantnegative effect."

Reverse transcription-quantitative real-time PCR (RT-qPCR) analysis demonstrated that SRC-3 mRNA levels were not affected by any SPOP (WT or mutant), which confirmed our prior finding that the impact of SPOP ${ }^{\mathrm{WT}}$ on SRC-3 expression is posttranslational (16).
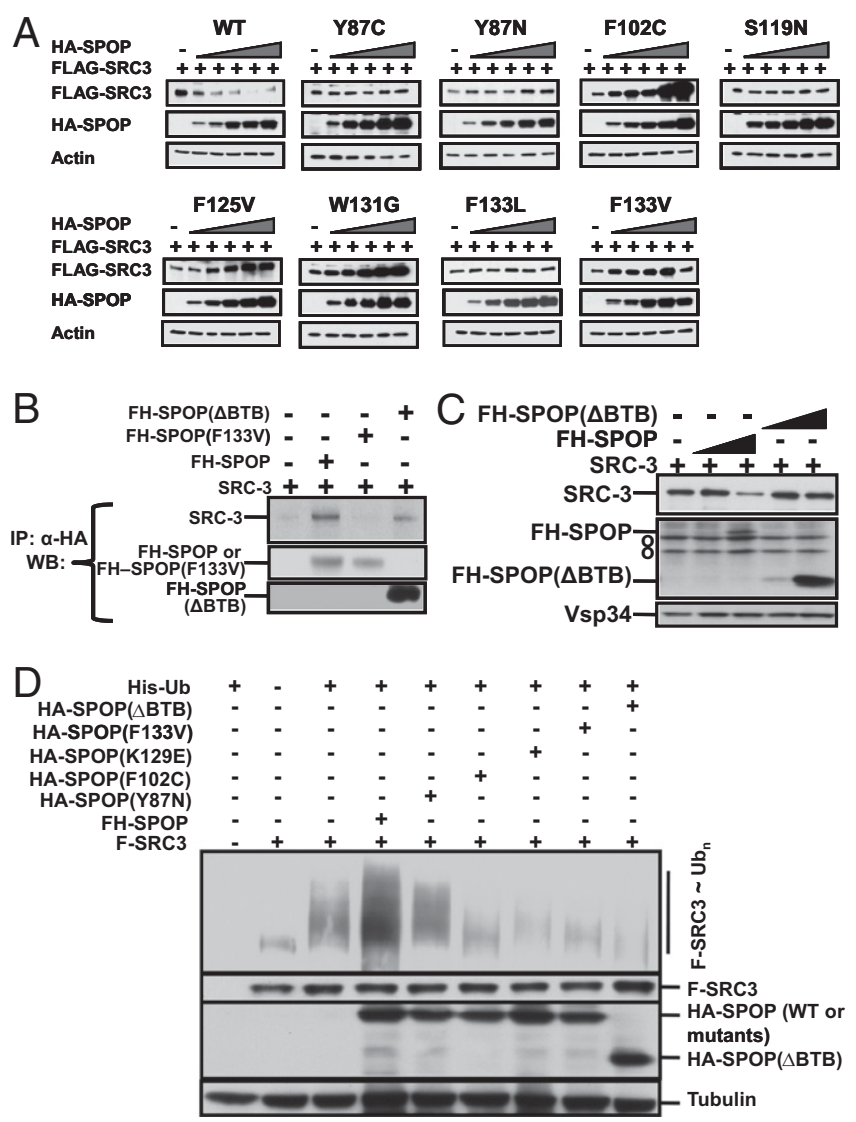

Fig. 2. SPOPWT, but not PC-associated SPOP mutants, promotes degradation of SRC-3 protein through a mechanism that involves ubiquitination and requires the SPOP BTB domain. (A) HEK293T cells were cotransfected with $0.6 \mu \mathrm{g} \mathrm{pCMV-FLAG} \mathrm{2B-SRC-3} \mathrm{and} \mathrm{different} \mathrm{amounts}(0,0.2,0.4,0.8,1.6$, and $2.0 \mu \mathrm{g})$ of pCDNA3.1-Hygro-HA-SPOP or each of the pcDNA3.1-HygroHA-SPOP mutants. After $72 \mathrm{~h}$, the FLAG-SRC-3 and HA-SPOPs expressed in these cells were analyzed by immunoblotting. Actin was used as a loading control. SPOPWT efficiently promoted degradation of SRC-3 in a dosedependent manner. None of the PC-associated SPOP mutants could promote degradation of SRC-3 protein. (B) SRC-3 interacts with SPOP or SPOP ( $\triangle \mathrm{BTB}$ ), but not PC-associated F133V mutant. 293T cells were transiently transfected with FLAG-tagged SRC-3, together with FLAG-HA-tagged SPOP, SPOP(F133V), or SPOP( $\triangle B T B)$. SPOP proteins were immunoprecipitated with anti-HA antibody and immunoblotted with anti-FLAG antibody. WB, Western blot. (C) SRC-3 degradation in PC cells is promoted by SPOP but not SPOP ( $\triangle B T B)$. PC-3 cells were transiently transfected with SRC-3 and different doses of FLAG-HA-tagged (FH)-SPOP or FH-SPOP( $\triangle B T B$ ), and subjected to immunoblotting with antibodies against SRC-3 or FLAG. Vsp34 served as loading control. 0 , nonspecific band. (D) PC-associated SPOP mutants are defective at promoting SRC-3 ubiquitination. 293T cells were transiently transfected with $3 \mu \mathrm{g}$ His-Ub and $5 \mu \mathrm{g}$ FLAG-SRC-3, together with $10 \mu \mathrm{g}$ FLAG-HA-tagged wild-type SPOP or HA-tagged SPOP mutants [PC-associated mutants or SPOP( $\triangle \mathrm{BTB})]$ for $48 \mathrm{~h}$, and then treated with proteasome in hibitor (20 $\mu \mathrm{M} \mathrm{MG132}$ for $2 \mathrm{~h}$ ). Cells were harvested under denaturing conditions. His-Ub-modified cellular proteins were purified by Ni-NTA agarose resin and subjected to SDS/PAGE. Ubiquitinated SRC-3 was detected by immunoblotting with anti-SRC-3 antibody (topmost panel). Cell extracts were also probed with antibodies against FLAG for F-SRC-3, HA for wild-type or SPOP mutants, or tubulin (loading control). 
SPOP ${ }^{W T}$, but Not Its PC-Associated Mutants, Promotes Ubiquitination of SRC-3 Protein Through a Mechanism That Requires the SPOP BTB Domain. We have previously reported that deletion of the 203 carboxyl-terminal amino acid residues from SPOP (amino acids 172-374, a region that includes amino acids 184-297, the BTB domain; Fig. $1 A$ ) abrogates its capacity to bind cullin 3 and promote degradation of SRC-3 (16). We now generated an expression vector encoding SPOP that lacks only amino acids 184-297, the $\mathrm{BTB}$ domain $[\operatorname{SPOP}(\triangle \mathrm{BTB})]$. As a result, $\operatorname{SPOP}(\triangle \mathrm{BTB})$ cannot bind cullin 3 . We found that, although $\operatorname{SPOP}(\triangle \mathrm{BTB})$ retains the capacity to bind SRC-3 (Fig. $2 B$ ), it cannot promote degradation of SRC-3 in prostate cancer PC-3 cells (Fig. $2 C$ ). For comparison, the PC-associated SPOP ${ }^{\mathrm{F} 133 \mathrm{~V}}$ cannot bind SRC-3 (Fig. 2B) and cannot promote its degradation (Fig. $2 C$ ).

These data suggest that SPOP-mediated degradation of SRC-3 requires the BTB domain-mediated recruitment to cullin 3 (which is necessary for substrate ubiquitination). Indeed, in agreement with our prior report (16), we confirmed that SPOP ${ }^{\mathrm{WT}}$ promotes SRC-3 ubiquitination (Fig. 2D). However, PC-associated SPOP mutants and $\operatorname{SPOP}(\triangle \mathrm{BTB})$ also failed to promote SRC-3 ubiquitination (Fig. 2D), thus providing an explanation for their inability to promote SRC-3 degradation. Of note, a subset of SPOP mutants decreased SRC-3 ubiquitination below baseline (i.e., no exogenous SPOP) levels, again suggesting a possible dominant-negative effect of these SPOP mutants on the function of endogenous (WT) SPOP.

PC-Associated SPOP Mutants Lack the Capacity of SPOPWT to Attenuate the Coactivator Function of SRC-3 on AR Transcriptional Activity. Because SRC-3 is an important coactivator of the transcriptional activity of AR, we next examined the impact of these PC-associated SPOP mutants on AR activity in vitro. First, we transiently expressed SPOP ${ }^{\mathrm{WT}}$ or SPOP mutants in HepG2 cells and quantified their capacity to modulate the impact of SRC-3 on AR transcriptional activity, using a reporter plasmid carrying a 6-kb fragment of the promoter/enhancer region of the prostate-specific antigen (PSA; gene name: kallikrein-related peptidase 3; KLK3) gene. This region harbors three functionally active AR-binding sites (androgen response elements; AREs) (22). As anticipated, we found that SRC-3 can robustly stimulate the transcriptional response of the $K L K 3$ gene promoter/enhancer to androgen. This effect is significantly attenuated by SPOP ${ }^{\mathrm{WT}}$, but not by the

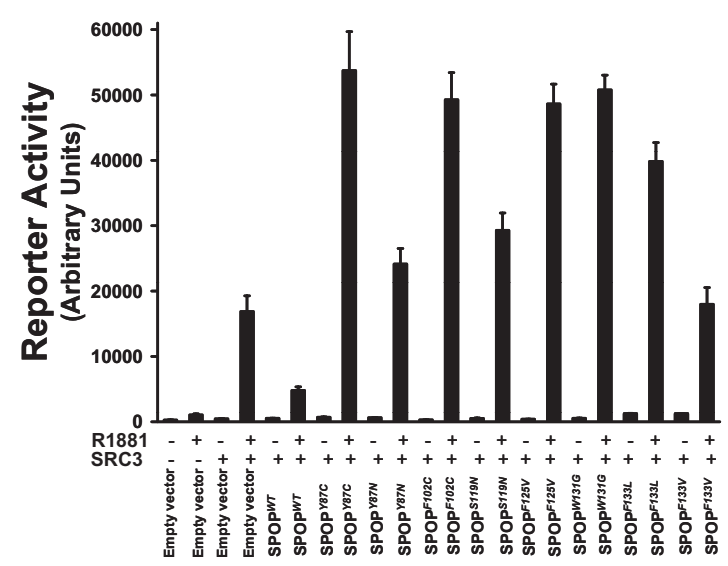

Fig. 3. PC-associated SPOP mutants lack the capacity of SPOPWT to attenuate the coactivator function of SRC-3 on AR transcriptional activity. HepG2 cells were transfected with expression vectors for AR, SRC-3, and SPOP (WT or mutant) or the corresponding empty vectors, as well as PSA61-Luc reporter vector (that carries the promoter and upstream enhancer region of the PSA gene). The cells were treated $24 \mathrm{~h}$ later with $10 \mathrm{nM}$ R1881 or vehicle (EtOH). Luciferase activity was determined $24 \mathrm{~h}$ later using the Promega Luciferase Assay Kit according to the manufacturer's protocols. Error bars represent SD.
PC-associated SPOP mutants (Fig. 3). Once again, a subset of mutants (including F102C, F125V, and W131G) increased AR transcriptional activity above baseline (i.e., no exogenous SPOP) levels, suggesting a possible gain-of-function dominant-negative effect of these SPOP mutants on the function of endogenous (WT) SPOP.

SPOP ${ }^{\mathrm{WT}}$, but Not Its PC-Associated Mutants, Binds SRC-3 and Suppresses Endogenous SRC-3 Protein Levels in PC Cells. To study the detailed biological functions of SPOP and its PC-associated mutants in PC cells, we transfected the PC cell line LNCaP-Abl with tetracyclineinducible expression vectors encoding for SPOP ${ }^{\mathrm{WT}}$ or each of the SPOP mutants and established stable cell lines under antibiotic selection. Endogenous SRC-3 protein in these PC cells could be coimmunoprecipitated with doxycycline-induced SPOP ${ }^{\mathrm{WT}}$, but not by mutant SPOP (Fig. $4 A$ ). Induction of SPOP ${ }^{\mathrm{WT}}$ with doxycycline potently suppressed endogenous SRC-3 protein levels. On the contrary, this suppressive effect was not seen upon induction of SPOP mutants by doxycycline (Fig. 4B).

SPOP ${ }^{\mathrm{WT}}$, but Not Its PC-Associated Mutants, Suppresses AR Transcriptional Activity and Is a Potent Tumor Suppressor in PC Cells. Because SRC-3 is an important coactivator of the transcriptional activity of $\mathrm{AR}$, we next explored the impact of SPOP ${ }^{\mathrm{WT}}$ and each of the PC-associated SPOP mutants on AR signaling in PC cells. RT-qPCR analysis for PSA $(K L K 3)$ gene expression levels in LNCaP-Abl PC cells demonstrated that doxycycline-induced expression of SPOPWT significantly suppressed the expression of this AR-dependent gene (suggesting suppression of AR transcriptional activity). However, this suppressive effect was attenuated in the case of mutant SPOPs (Fig. 5A). Similar results were seen (Fig. S2) for the expression of IGF1 [a gene known to be SRC-3-dependent in PC cells (8)] and FKBP5 (also known to be AR-dependent in PC cells). Finally, using the 3-(4,5-dimethylthiazol-2-yl)-2,5-diphenyltetrazolium bromide (MTT) assay, we examined the impact of doxycyclineinduced WT and mutant SPOPs on the proliferative rate of LNCaP-Abl PC cells. We documented a tumor suppressor effect for SPOP ${ }^{\mathrm{WT}}$ in LNCaP-Abl PC cells, which was attenuated in the case of mutant SPOPs (Fig. $5 B$ ).

Endogenous SPOP Regulates SRC-3 Protein Expression and Suppresses AR Transcriptional Activity in PC Cells. Finally, we explored whether endogenous SPOP regulates SRC-3 expression and AR transcriptional activity in a panel of six commonly used AR-positive PC cell lines (three androgen-dependent and three androgenindependent). All six cell lines had been previously found, by Sanger sequencing, to lack mutations in the SPOP-coding sequence. We found that silencing SPOP via siRNA resulted in increased SRC-3 protein levels and increased expression of the AR-target gene PSA (KLK3; Fig. S3). These data confirm that SPOP is an endogenous regulator of SRC-3 protein turnover and AR activity in PC cells and provide a possible explanation for the fact that it is the most commonly mutated gene in PC.

\section{Discussion}

Recent exome-sequencing studies have identified SPOP as the gene most commonly affected by somatic nonsynonymous point mutations in PC (19-21). However, the role of these SPOP mutants in PC pathophysiology was unknown. We have previously characterized a Cul3-based, SPOP-dependent complex as an E3 ubiquitin ligase that promotes ubiquitination and posttranslational turnover of SRC-3 protein (16). As SRC-3 can potently promote AR transcriptional activity and pleiotropic oncogenic signaling necessary for cancer cell proliferation, survival, metabolism, and metastasis, we hypothesized that SRC-3 could be one of the major SPOP substrates mediating the effect of mtSPOP in PC and investigated the impact of WT and mtSPOP on SRC expression and function. Overexpression of SPOP ${ }^{\mathrm{WT}}$ potently promoted the 


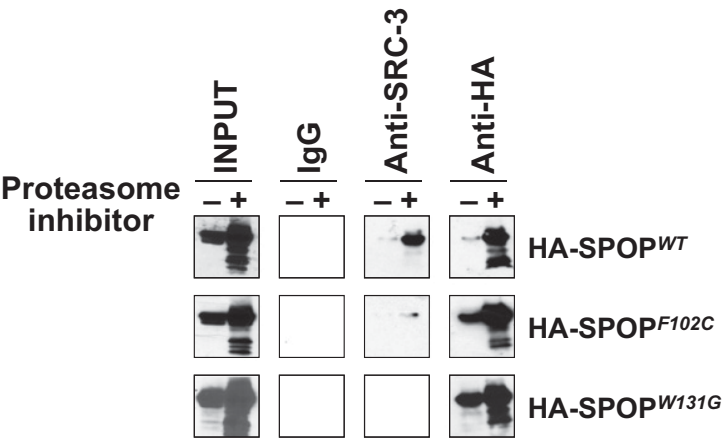

B
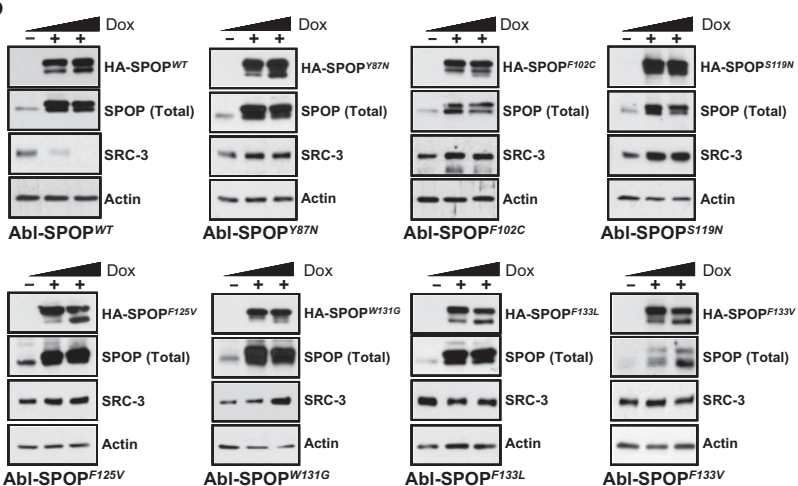

Fig. 4. $S P O P^{W T}$, but not its $P C$-associated mutants, binds SRC-3 and suppresses endogenous SRC-3 protein levels in PC cells. (A) LNCaP-Abl PC cells stably transfected with tetracycline-inducible constructs encoding for SPOPWT or PC-associated SPOP mutants were treated with $200 \mathrm{ng} / \mathrm{mL}$ doxycycline for a total of $48 \mathrm{~h}$. During the last $24 \mathrm{~h}$ of the incubation, the cells were also treated with a proteasome inhibitor (PS-341, $250 \mathrm{nM}$ ) or vehicle (DMSO). The cells were then lysed in lysis buffer, as in Fig. 1B. Immunoprecipitation was performed using antibodies against endogenous SRC-3 or HA or control IgG. In cells treated with the proteasome inhibitor, SRC-3 could coprecipitate SPOPWT, whereas its capacity to bind the PC-associated SPOP mutants was found to be impaired. (B) LNCaP-Abl (Abl) PC cells stably transfected with tetracycline-inducible constructs encoding for SPOPWT or each of the PC-associated SPOP mutants were treated with 0,50 , or $500 \mathrm{ng} / \mathrm{mL}$ doxycycline (Dox) for $72 \mathrm{~h}$, lysed, and immunoblotted using antibodies against the HA tag (recognizing only the transfected WT or mutant SPOP), SPOP itself (recognizing both endogenous and transfected SPOPs), SRC-3, and actin.

degradation of SRC-3 protein, but not SRC-1 or SRC-2 protein. All PC-associated SPOP mutants tested failed to promote SRC-3 ubiquitination and protein degradation. We further documented the physical interaction of SPOP ${ }^{\mathrm{WT}}$ with SRC-3 (but not SRC-1 or SRC-2), and this interaction was abolished in these PC-associated SPOP mutants. In PC cells, SPOP ${ }^{\mathrm{WT}}$ suppresses SRC-3 protein expression, cell proliferation, and AR transcriptional activity, whereas this effect is abolished or significantly attenuated by the PC-associated SPOP mutations.

We have previously reported that the SPOP N-terminal MATHcontaining domain directly binds to SRC-3, whereas the SPOP C-terminal BTB-containing domain directly binds to $\mathrm{Cul3}$ (16). A five-residue $\varphi-\pi$-S-S/T-S/T ( $\varphi$, nonpolar; $\pi$, polar) SPOP-binding consensus motif has been described (18), and a similar sequence is present in SRC-3 upon phosphorylation of SRC-3 at S102 (16). This site is phosphorylated by casein kinase I $\varepsilon$ (16). Therefore, SPOP can act as an adaptor for this Cul3-based ubiquitin ligase complex and target the SRC-3 protein for degradation in a phosphorylation-dependent manner (16). We now demonstrate that this effect is restricted to SRC-3, and not present in SRC-1 or SRC-2. Importantly, this tumor suppressor effect of SPOP is abolished or significantly attenuated by the human PC-associated SPOP mutations that cluster in the MATH domain. These data highlight SRC-3 as an effector of the SPOP mutations in PC.

SRC-3 (AIB1/ACTR/NCOA3) is a key pleiotropic master regulator of transcription factor activity necessary for cancer cell proliferation, survival, metabolism, and metastasis. SRC-3 overexpression and overactivation occur in numerous human cancers, due to a variety of genomic, transcriptional, and posttranslational mechanisms, and are associated with poor clinical outcomes and resistance to therapy. Not surprisingly, SRC-3 protein turnover is controlled at multiple levels. Inactive, steady-state SRC-3 can be degraded in a ubiquitin- and ATP-independent manner by the $\mathrm{REG} \gamma$ proteasome (23). When activated by phosphorylation at a number of important sites (including the essential SRC-3 phosphodegron residues S101 and S102), SRC-3 stability is mainly controlled by the SPOP-Cul3-Rbx1 ubiquitin ligase complex, in a mechanism regulated by the balance between phosphorylation at S102 [e.g., by casein kinase I $\varepsilon$ (16)] and dephosphorylation [e.g., by PP1 (24)]. Finally, upon recruitment to nuclear receptor transcription complexes on gene promoters, GSK3-dependent phosphorylation of S505 and S509 promotes SCF(Fbw7 1 )dependent SRC-3 ubiquitination, linking transcription and SRC-3
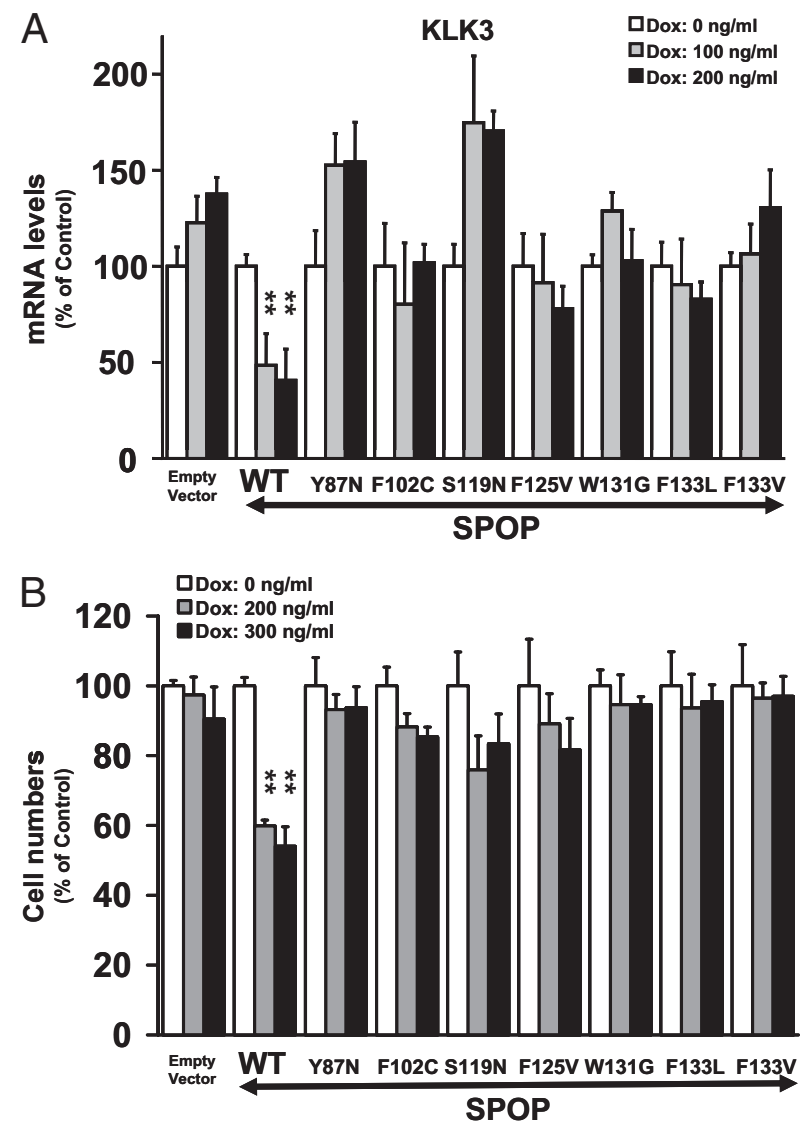

Fig. 5. SPOPWT, but not its $P C$-associated mutants, suppresses endogenous AR transcriptional activity and is a potent tumor suppressor in PC cells. $(A)$ $\mathrm{Abl}$ cells stably transfected with tetracycline-inducible constructs encoding for SPOP ${ }^{W T}$ or each of the PC-associated SPOP mutants were treated with 0,100 , or $200 \mathrm{ng} / \mathrm{mL}$ doxycycline for $48 \mathrm{~h}$. Expression of the AR-dependent gene KLK3 (PSA) was quantified by RT-qPCR. ** $P<0.01$ for doxycyclinetreated versus vehicle-treated cells. $(B)$ MTT assay was used to determine the proliferation of LNCaP-Abl cells when SPOPWT or SPOP mutants were expressed in these cells. Cells were treated with 0,200 , or $300 \mathrm{ng} / \mathrm{mL}$ doxycycline for $6 \mathrm{~d}$. Experiments were repeated at least three times, with each experimental condition repeated at least in quadruplicate per experiment. $* \star P<0.01$ for doxycycline-treated versus vehicle-treated cells. 
degradation (25). This highly complex network of three posttranslational modification mechanisms that control the stability and function of SRC-3 highlights its importance as a potent master regulator of gene expression.

Of note, our experimental results have raised the possibility that certain SPOP mutants (including F102C and W131G) may exert a "gain-of-function" oncogenic effect by increasing SRC-3 protein levels and AR transcriptional activity above baseline (i.e., no exogenous SPOP) levels. This phenomenon, which could be attributed to a putative dominant-negative effect on the function of SPOP ${ }^{\mathrm{WT}}$, may acquire particular importance because SPOP mutations are heterozygous in prostate cancer specimens. However, it should be acknowledged that there was some variability in the behavior of these SPOP mutants across our wide spectrum of experimental models and readouts; that is, the same SPOP mutant did not always behave as a dominant-negative across all models and assays (although all SPOP mutants were consistently and universally found to have attenuated tumor suppressor properties compared with SPOP ${ }^{\mathrm{WT}}$ ). Such variability is perhaps attributable to underlying variation in our model systems or other experimental conditions. As a result, the identification and delineation of a unifying model for the dominant-negative function of these SPOP mutants will require additional detailed studies. In any case, our data hint at possible functional differences between the various SPOP mutants regarding their oncogenic potential and even prognostic significance, which, obviously, will need to be validated in clinically annotated human prostate cancer specimens.

Obviously, SPOP is known to bind and promote the ubiquitination of other substrates, including the death domain-associated protein Daxx (26), the phosphatase Puc, the transcriptional regulator $\mathrm{Ci} / \mathrm{Gli}$ (27), the variant histone MacroH2A (18), and several others. Therefore, SPOP mutations could possibly affect other signaling pathways beyond SRC-3/AR, and our data do not exclude such a hypothesis. However, we have provided strong evidence that the transcriptional coactivator SRC-3 and the nuclear hormone receptor $\mathrm{AR}$, both critically important for $\mathrm{PC}$ pathophysiology and resistance to therapy, are downstream effectors of SPOP. As the PC-associated SPOP mutations are predicted to be loss-of-function, they may not be directly druggable for therapeutic purposes. A targeted-treatment approach to SPOP mutations in PC could be to inhibit its downstream substrates that accumulate in the absence of SPOP function. Our study identifies SRC-3 as a mediator of the oncogenic effects of mtSPOP and highlights the need to develop inhibitors of the p160 SRCs.

In summary, we have demonstrated that SPOP ${ }^{\mathrm{WT}}$ plays a critical tumor suppressor role in PC cells by promoting the ubiquitination and proteasomal degradation of SRC-3 protein and, thus, suppressing AR transcriptional activity. This tumor suppressor effect is abrogated by the PC-associated SPOP mutations. Our data provide a possible explanation for the impact of the recurrent somatic heterozygous missense mutations in the SPOP MATH domain that were recently identified in a subset of human PCs (19-21), and highlight again the potential of SRC-3 as a therapeutic target in PC.

\section{Materials and Methods}

Cell Culture. Human embryonic kidney 293T cells (American Type Culture Collection) were cultured in DMEM high-glucose (Invitrogen) with 10\% (vol/ vol) FBS (Invitrogen) in a $5 \%$ (vol/vol) $\mathrm{CO}_{2}$ incubator at $37^{\circ} \mathrm{C}$. LNCaP-Abl cells, a PC cell line derived from LNCaP cells via selection in androgen-depleted medium (supplemented with 10\% charcoal-stripped FBS; CSS) (28), was a generous gift from Zoran Culig (University of Innsbruck, Innsbruck, Austria) and maintained in RPMI1640 (Invitrogen) supplemented with 10\% CSS Doxycycline-inducible Abl stable transfectants were maintained in RPMI1640 supplemented with $10 \%$ tetracycline-free FBS (Atlanta Biologicals, Inc.) and $300 \mu \mathrm{g} / \mathrm{mL}$ of the selection antibiotic G-418 (Invitrogen). Human hepatoblastoma HepG2 cells (American Type Culture Collection) were maintained in $5 \% \mathrm{CO}_{2}$ at $37{ }^{\circ} \mathrm{C}$ in Eagle's MEM (Invitrogen) with $10 \%$ FBS (Invitrogen).
Expression Constructs. Using Phusion High-Fidelity DNA Polymerase (New England BioLabs) and $\mathrm{PCR}$ primers containing an $\mathrm{HA}$ tag at the $\mathrm{N}$ terminus, an expression construct of HA-tagged SPOP (pcDNA3.1-HA-SPOP, wild-type) was constructed by insertion of the PCR-amplified SPOP CDNA-coding sequence into mammalian expression vector pcDNA3.1 Hygro (+) (Invitrogen). In vitro site-directed mutagenesis was used to obtain the HA-tagged SPOP mutants by two PCR amplifications using PCDNA3.1-HA-SPOP as the template. The PCR-amplified DNAs coding for mutated SPOPs were inserted into pCDNA3.1 Hygro (+) (Invitrogen) to generate the corresponding mammalian expression vectors: pcDNA3.1-HA-SPOP ${ }^{F 102 C}$, pcDNA3.1-HA-SPOP ${ }^{F 125 V}$, pcDNA3.1HA-SPOP ${ }^{F 133 V}$, pCDNA3.1-HA-SPOP ${ }^{F 133 L}$, pCDNA3.1-HA-SPOP ${ }^{Y 87 N}$, pCDNA3.1HA-SPOP ${ }^{\text {Y7C }}$, ${ }^{\prime}$ PCDNA3.1-HA-SPOP ${ }^{W 131 G}$, and pCDNA3.1-HA-SPOP'S119N

Lentiviral, doxycycline-inducible expression vectors for SPOPWT and its PCassociated mutants, plnducer-HA-SPOP, plnducer-HA-SPOP ${ }^{F 102 C}$, plnducerHA-SPOP $^{F 125 V}$, plnducer-HA-SPOP ${ }^{F 133 V}$, plnducer-HA-SPOP ${ }^{F 133 L}$, plnducerHA-SPOP ${ }^{Y 87 N}$, and plnducer-HA-SPOP ${ }^{\text {Wi31G }}$, were generated by the Gateway cloning technique using the PENTR/TEV/D-TOPO Cloning Kit (Invitrogen), Gateway LR Clonase Enzyme (Invitrogen), and lentiviral vector plnducer 20 [a kind gift from T. Westbrook, Baylor College of Medicine (29)]. For all constructs, correct insertion was confirmed with Sanger sequencing. The PCR primer sequences used to generate these constructs are listed in Table S1.

Lentivirus Production and Establishment of Prostate Cancer Cell Lines with Doxycycline-Inducible Expression of SPOP ${ }^{\text {WT }}$ or Each of SPOP Mutants. The plnducer-HA-SPOP ${ }^{W T}$ or -HA-SPOP ${ }^{m t}$ vectors were cotransfected with lentiviral packaging plasmid vectors (pHDM-HsDM2, pHDM-VsVg, pHDM-tat1,6, and PRC-CMV-Polli; generous gifts from T. Westbrook) into 293T cells as previously described (29). Two days after the transfection, the virus-containing medium was collected from each transfection and sterilized by passing through a $0.45-\mu \mathrm{m}$ low-protein-binding filtration cartridge. The virus particles were directly used to infect LNCaP-Abl cells in the presence of polybrene $(4 \mu \mathrm{g} / \mathrm{mL})$ for $48 \mathrm{~h}$ before $600 \mu \mathrm{g} / \mathrm{mL}$ G-418 (Invitrogen) was introduced for $3 \mathrm{wk}$ to select the stable cell lines. Afterward, the obtained stable cell lines LNCaP-Abl-SPOP ${ }^{W T}$, LNCaP-AbI-SPOP ${ }^{F 102 C}$, LNCaPAbl-SPOP $^{F 125 V}$, LNCaP-Abl-SPOP ${ }^{F 133 V}$, LNCaP-Abl-SPOP ${ }^{F 133 L}$ LNCaP-Abl-SPOP $^{\text {Y87N }}$, and LNCaP-Abl-SPOP ${ }^{W 131 G}$ were maintained in RPMI1640 culture medium supplemented with $10 \%$ tetracycline-free FBS (Atlanta Biologicals, Inc.) and $\mathrm{G}-418(300 \mu \mathrm{g} / \mathrm{mL})$ before analysis.

Coimmunoprecipitation. Coimmunoprecipitation was conducted according to a standard protocol described previously (30) with minor modification. Briefly, HEK293T cells were transfected with HA-SPOP (WT or each individual mutant) or FLAG-tagged SRC (-1, -2 , or -3) expression vectors (pSG5-SRC-1FLAG, pSG5-SRC-2-FLAG, or pCMV-tag2B-SRC-3, respectively). Two days after transfection, the cells were lysed in lysis buffer $(50 \mathrm{mM}$ Tris. $\mathrm{HCl}, \mathrm{pH} 8.0,150 \mathrm{mM}$ $\mathrm{NaCl}, 1 \%$ Nonidet $\mathrm{P}-40$ ) containing protease inhibitor mixture (Sigma). The expressed HA-SPOPs and SRC-3 in 293T cell lysates were determined and quantified by immunoblotting and DC protein quantification kit (Bio-Rad). For coimmunoprecipitation analysis of HA-SPOPs with SRC-3, cell lysates containing an approximately equal amount of each expressed HA-SPOP (WT or mutant) and SRC-3 protein were combined. The HA-SPOP/SRC-3 cell lysate mix was incubated with anti-FLAG M2 antibody (Sigma) overnight at $4{ }^{\circ} \mathrm{C}$ with constant rotation before protein A Dynabeads (Invitrogen) were added to collect the immunocomplex. The beads were washed four times with lysis buffer and boiled in Laemmli sample-loading buffer for $10 \mathrm{~min}$ to elute the precipitated proteins. The supernatants were separated by SDS/PAGE, and immunoblotting was used to detect HA-SPOPs and FLAG-SRC-3. The input was loaded at 1/10 of the total lysate amount subjected to each immunoprecipitation experiment.

SDS/PAGE and Immunoblotting. The protein lysates were separated by SDS/ PAGE and transferred onto nitrocellulose membranes. Immunoblotting was conducted by using protein-specific antibodies as previously described (31, 32). Briefly, the membranes were first blocked with 5\% Blocking Reagent (BioRad) in phosphate buffered saline with $0.05 \%$ Tween 20 (PBS-T) before incubation with antibodies overnight. For co-IP Western blotting analysis using anti-FLAG-HRP and anti-HA-HRP, after washes with PBS-T buffer the membranes were imaged on X-ray films using SuperSignal Western Blotting Kits according to the manual instructions (Thermo Fisher Scientific). Otherwise, the PBS-T-washed membranes were further incubated with anti-lgG-HRP (Santa Cruz) and imaged as indicated above. The antibodies used in Western blotting were mouse anti-HA (Roche), monoclonal mouse anti-FLAG M2 (Sigma), anti-SPOP (Abcam), monoclonal rabbit anti-SRC-3 (Cell Signaling), rabbit anti-SRC-1 (Santa Cruz), rabbit anti-GRIP/SRC-2 (Santa Cruz), anti$\beta$-actin (Sigma), mouse anti-FLAG-HRP (Sigma), mouse anti-HA-HRP (Roche), anti-rabbit IgG-HRP (Sigma), and anti-mouse IgG-HRP (Sigma). 
Reverse Transcription-Quantitative Real-Time PCR. Reverse transcription and PCR reactions were performed with a TaqMan RNA-to-CT 1-Step Kit (Applied Biosystems) using a StepOnePlus Real-Time PCR System (Applied Biosystems). Results were normalized to the same amount of total RNA. The primers and probes used for KLK3, IGF1, SRC-3, and FKBP5 transcripts are listed in Table S2.

Reporter Assay Analysis. Human hepatoblastoma HepG2 cells (American Type Culture Collection) were maintained in $5 \% \mathrm{CO}_{2}$ at $37{ }^{\circ} \mathrm{C}$ in Eagle's MEM (Invitrogen). Cells were plated at $1 \times 10^{5}$ cells per well in 24-well tissue culture plates and transfected with $3 \mathrm{ng}$ of pCMV5-AR, $100 \mathrm{ng}$ of the PSA61luc reporter vector [that carries a 6 -kb fragment of the PSA promoter/enhancer region, harboring three functional AREs, inserted upstream of the reporter gene (22)], $100 \mathrm{ng}$ of pCR3.1-SRC-3 (or empty vector), and $100 \mathrm{ng}$ of pCDNA3.1 plasmids encoding WT SPOP or each mutant SPOP (or empty vector), using Lipofectamine LTX and Plus Transfection Reagent (Invitrogen). Twenty-four hours after transfection, synthetic androgen (R1881) or vehicle was added to the appropriate wells (final concentration of R1881, $10 \mathrm{nM}$ ). Luciferase activity was determined $24 \mathrm{~h}$ later using the Promega Luciferase Assay Kit according to the manufacturer's protocols.

In Vivo Ubiquitination Assay. For analysis of the ubiquitination of SRC-3 in cells, 293T cells were transiently cotransfected with His-Ub, FLAG-tagged SRC-3, and either FLAG-HA-tagged wild-type SPOP or HA-tagged SPOP mutants. Fortyeight hours posttransfection, cells were treated with $20 \mu \mathrm{M}$ MG132 for $2 \mathrm{~h}$ and harvested under denaturing conditions, as described (33). His-Ub-conjugated cellular proteins were purified by Ni-NTA agarose resin. Ubiquitinated SRC-3 was detected using SDS/PAGE and immunoblotting with the anti-SRC-3 antibody.

1. de Bono JS, et al.; COU-AA-301 Investigators (2011) Abiraterone and increased survival in metastatic prostate cancer. N Engl J Med 364(21):1995-2005.

2. Scher HI, et al.; AFFIRM Investigators (2012) Increased survival with enzalutamide in prostate cancer after chemotherapy. N Engl J Med 367(13):1187-1197.

3. Mitsiades N, et al. (2012) Distinct patterns of dysregulated expression of enzymes involved in androgen synthesis and metabolism in metastatic prostate cancer tumors. Cancer Res 72(23):6142-6152.

4. Agoulnik IU, et al. (2006) Androgens modulate expression of transcription in termediary factor 2 , an androgen receptor coactivator whose expression level correlates with early biochemical recurrence in prostate cancer. Cancer Res 66(21): 10594-10602.

5. Taylor BS, et al. (2010) Integrative genomic profiling of human prostate cancer. Cancer Cell 18(1):11-22.

6. Xu J, Wu RC, O'Malley BW (2009) Normal and cancer-related functions of the p160 steroid receptor co-activator (SRC) family. Nat Rev Cancer 9(9):615-630.

7. Zhou HJ, et al. (2005) SRC-3 is required for prostate cancer cell proliferation and survival. Cancer Res 65(17):7976-7983.

8. Yan J, et al. (2006) Steroid receptor coactivator-3 and activator protein-1 coordinately regulate the transcription of components of the insulin-like growth factor/AKT signaling pathway. Cancer Res 66(22):11039-11046.

9. Yan J, et al. (2008) Steroid receptor coactivator-3/AIB1 promotes cell migration and invasiveness through focal adhesion turnover and matrix metalloproteinase expression. Cancer Res 68(13):5460-5468.

10. O'Malley BW, Kumar R (2009) Nuclear receptor coregulators in cancer biology. Cancer Res 69(21):8217-8222.

11. Agoulnik IU, Weigel NL (2009) Coactivator selective regulation of androgen receptor activity. Steroids 74(8):669-674.

12. Agoulnik IU, et al. (2005) Role of SRC-1 in the promotion of prostate cancer cell growth and tumor progression. Cancer Res 65(17):7959-7967.

13. Zhou G, Hashimoto Y, Kwak I, Tsai SY, Tsai MJ (2003) Role of the steroid receptor coactivator SRC-3 in cell growth. Mol Cell Biol 23(21):7742-7755.

14. Demichelis F, et al. (2009) Distinct genomic aberrations associated with ERG re arranged prostate cancer. Genes Chromosomes Cancer 48(4):366-380.

15. Grasso CS, et al. (2012) The mutational landscape of lethal castration-resistant prostate cancer. Nature 487(7406):239-243.

16. Li C, et al. (2011) Tumor-suppressor role for the SPOP ubiquitin ligase in signal-dependent proteolysis of the oncogenic co-activator SRC-3/AIB1. Oncogene 30(42):4350-4364.
MTT Assay. Cells were plated in 24-well plates in medium containing $10 \%$ FBS and allowed to adhere for $24 \mathrm{~h}$. Then, drugs were added, and the cells were incubated for 96 more hours. Cell numbers were quantified by MTT (SigmaAldrich) as previously described $(31,32)$, and expressed as a percentage of the value of control wells. All experiments were repeated at least three times, with each experimental condition repeated at least in quadruplicate per experiment.

Statistical Analysis. Each experiment was repeated at least three times. Fold changes in mRNA levels (RT-qPCR), reporter assay activity, or cell number (absorbance in MTT assay) were compared between differently treated samples (with or without doxycycline treatment) using one-way ANOVA. In all analyses, $P<0.05$ was considered statistically significant.

ACKNOWLEDGMENTS. We acknowledge the joint participation by The Adrienne Helis Malvin Medical Research Foundation through its direct engagement in the continuous active conduct of medical research in conjunction with Baylor College of Medicine. This work was supported by the Prostate Cancer Foundation (C.E.B., F.D., M.A.R., P.Z., B.W.O., and N.M.) National Institute of Child Health and Human Development Grant 8818 (to B.W.O.); Conquer Cancer Foundation of the American Society of Clinical Oncology Young Investigator and Career Development Awards (both to N.M.); and a Pilot/Feasibility Program grant of the Diabetes and Endocrinology Research Center (P30DK079638) at Baylor College of Medicine (to N.M.). N.M. is a Dan L. Duncan Scholar, a Caroline Wiess Law Scholar, and a member of The Dan L. Duncan Cancer Center (supported by National Cancer Institute Cancer Center Support Grant P30CA125123) and the Center for Drug Discovery at Baylor College of Medicine.

17. Bunce MW, Boronenkov IV Anderson RA (2008) Coordinated activation of the nuclear ubiquitin ligase Cul3-SPOP by the generation of phosphatidylinositol 5-phosphate. $J$ Biol Chem 283(13):8678-8686.

18. Zhuang $M$, et al. (2009) Structures of SPOP-substrate complexes: Insights into molecular architectures of BTB-Cul3 ubiquitin ligases. Mol Cell 36(1):39-50.

19. Berger MF, et al. (2011) The genomic complexity of primary human prostate cancer. Nature 470(7333):214-220.

20. Barbieri $\mathrm{CE}$, et al. (2012) Exome sequencing identifies recurrent SPOP, FOXA1 and MED12 mutations in prostate cancer. Nat Genet 44(6):685-689.

21. Kan Z, et al. (2010) Diverse somatic mutation patterns and pathway alterations in human cancers. Nature 466(7308):869-873.

22. Cleutjens KB, et al. (1997) An androgen response element in a far upstream enhancer region is essential for high, androgen-regulated activity of the prostate-specific an tigen promoter. Mol Endocrinol 11(2):148-161.

23. Li X, et al. (2006) The SRC-3/AIB1 coactivator is degraded in a ubiquitin- and ATPindependent manner by the REGgamma proteasome. Cell 124(2):381-392.

24. Li C, et al. (2008) Essential phosphatases and a phospho-degron are critical for regulation of SRC-3/AIB1 coactivator function and turnover. Mol Cell 31(6):835-849.

25. Wu RC, Feng Q, Lonard DM, O'Malley BW (2007) SRC-3 coactivator functional lifetime is regulated by a phospho-dependent ubiquitin time clock. Cell 129(6):1125-1140.

26. Kwon JE, et al. (2006) BTB domain-containing speckle-type POZ protein (SPOP) serves as an adaptor of Daxx for ubiquitination by Cul3-based ubiquitin ligase. $J$ Biol Chem 281(18):12664-12672.

27. Zhang Q, et al. (2009) Multiple Ser/Thr-rich degrons mediate the degradation of $\mathrm{Ci} / \mathrm{G}$ by the Cul3-HIB/SPOP E3 ubiquitin ligase. Proc Natl Acad Sci USA 106(50):21191-21196.

28. Culig Z, et al. (1999) Switch from antagonist to agonist of the androgen recepto bicalutamide is associated with prostate tumour progression in a new model system. Br J Cancer 81(2):242-251.

29. Meerbrey KL, et al. (2011) The pINDUCER lentiviral toolkit for inducible RNA interference in vitro and in vivo. Proc Natl Acad Sci USA 108(9):3665-3670.

30. Bonifacino JS, Dell'Angelica EC, Springer TA (2001) Immunoprecipitation. Curr Protoc $\mathrm{Mol}$ Biol chap. 10, unit 10.16

31. Mitsiades N, et al. (2011) Genotype-dependent sensitivity of uveal melanoma cell lines to inhibition of B-Raf, MEK, and Akt kinases: Rationale for personalized therapy. Invest Ophthalmol Vis Sci 52(10):7248-7255.

32. Mitsiades CS, et al. (2007) Targeting BRAFV600E in thyroid carcinoma: Therapeutic implications. Mol Cancer Ther 6(3):1070-1078.

33. Laney JD, Hochstrasser M (2011) Analysis of protein ubiquitination. Curr Protoc Protein Sci chap. 14, unit 14.15 . 